\title{
L-Band RFI as Experienced During Airborne Campaigns in Preparation for SMOS
}

\author{
Skou, Niels; Misra, Sidharth; Balling, Jan E.; Kristensen, Steen Savstrup; Søbjærg, Sten Schmidl
}

Published in:

I E E E Transactions on Geoscience and Remote Sensing

Link to article, DOI:

10.1109/TGRS.2009.2031637

Publication date:

2010

Document Version

Publisher's PDF, also known as Version of record

Link back to DTU Orbit

Citation (APA):

Skou, N., Misra, S., Balling, J. E., Kristensen, S. S., \& Søbjærg, S. S. (2010). L-Band RFI as Experienced During Airborne Campaigns in Preparation for SMOS. I E E E Transactions on Geoscience and Remote Sensing, 48(3), 1398-1407. https://doi.org/10.1109/TGRS.2009.2031637

\section{General rights}

Copyright and moral rights for the publications made accessible in the public portal are retained by the authors and/or other copyright owners and it is a condition of accessing publications that users recognise and abide by the legal requirements associated with these rights.

- Users may download and print one copy of any publication from the public portal for the purpose of private study or research.

- You may not further distribute the material or use it for any profit-making activity or commercial gain

- You may freely distribute the URL identifying the publication in the public portal 


\title{
L-Band RFI as Experienced During Airborne Campaigns in Preparation for SMOS
}

\author{
Niels Skou, Fellow, IEEE, Sidharth Misra, Jan E. Balling, Steen Savstrup Kristensen, and \\ Sten Schmidl Søbjærg, Member, IEEE
}

\begin{abstract}
In support of the European Space Agency Soil Moisture and Ocean Salinity (SMOS) mission, a number of soil moisture and sea salinity campaigns, including airborne L-band radiometer measurements, have been carried out. The radiometer used in this context is fully polarimetric and has built-in radio-frequency-interference (RFI)-detection capabilities. Thus, the instrument, in addition to supplying L-band data to the geophysicists, also gave valuable information about the RFI environment. Campaigns were carried out in Australia and in a variety of European locations, resulting in the largest and most comprehensive data set available for assessing RFI at L-band. This paper introduces the radiometer system and how it detects RFI using the kurtosis method, reports on the percentage of data that are typically flagged as being corrupted by RFI, and gives a hint about geographical distribution. Also, examples of polarimetric signatures are given, and the possibility of detecting RFI using such data is discussed.
\end{abstract}

Index Terms-Kurtosis, L-band, microwave, radio-frequency interference (RFI), radiometer.

\section{INTRODUCTION}

$\mathbf{R}$ ADIOMETER signals received in the L- and C-bands are particularly susceptible to man-made radio-frequency interference (RFI) due to many active services. RFI detection and possible mitigation have become an important concern, as both European Space Agency (ESA) and National Aeronautics and Space Administration will soon launch the Soil Moisture and Ocean Salinity (SMOS) [1] and Aquarius [2] satellite missions, respectively, to measure sea-surface salinity and soil moisture by means of L-band radiometry.

Little is known about the RFI situation when performing L-band radiometer measurements from space because no L-band radiometer mission has been launched since Skylab in the 1970s-a mission that did not report any RFI issues. In [3], a global survey of RFI in the 6.9- and 10.7-GHz Advanced Microwave Scanning Radiometer-Earth Observing System (AMSR-E) channels is presented. RFI is widely experienced. However, it is striking to observe the geographical distribution of the RFI. The observed RFI at $6.9 \mathrm{GHz}$ is most densely concentrated in the U.S., Japan, and the Middle East and is sparser in Europe. At $10.7 \mathrm{GHz}$, the RFI is concentrated

Manuscript received February 24, 2009; revised July 2, 2009. First published November 3, 2009; current version published February 24, 2010. This work was supported by the European Space Agency.

N. Skou, J. E. Balling, S. S. Kristensen, and S. S. Søbjærg are with the National Space Institute, Technical University of Denmark, Lyngby 2800, Denmark (e-mail: ns@space.dtu.dk; jeb@space.dtu.dk; ssk@space.dtu.dk; sss@space.dtu.dk).

S. Misra is with the Department of Atmospheric, Oceanic, and Space Sciences, University of Michigan, Ann Arbor, MI 48109-2143 USA (e-mail: samisra@umich.edu).

Digital Object Identifier 10.1109/TGRS.2009.2031637 mostly in England, Italy, and Japan. This, of course, does not prove anything about the situation at L-band, but bearing in mind the dense usage of the frequency spectrum around L-band for active services, we must expect RFI to be a problem of some magnitude. Early-warning air-defense radars and air-traffic-control radars are expected to be a primary source of L-band RFI [4]. We might also expect to find substantial regional differences according to the AMSR-E experiences.

Presently, the discussions about the potential threats to the forthcoming L-band missions must be based on experience from airborne campaigns, as well as expectations. This paper will report on RFI as experienced during the range of so-called CoSMOS campaigns carried out in the 2005-2008 period in support of the SMOS mission. Other campaign experiences are reported in [5].

It must be emphasized that the RFI that has been detected and dealt with in this paper is the RFI as received by one particular radiometer (EMIRAD-2) with its filter properties and as detected using one particular detection method (kurtosis) with its advantages and shortcomings. Thus, the results cannot be taken as an absolute truth, and another radiometer with other detection methods (see, for example, [6] and [7]) might give slightly different results. General tendencies and conclusions can, however, be made based on the present specific data set.

\section{About Kurtosis, Detection OF RFI, AND MITIGATION}

The microwave radiometer generally detects natural thermal emissions, as well as thermal noise of the hardware. These signals are random processes, and hence, the voltage has a Gaussian distribution. The kurtosis algorithm for RFI detection discussed in [8] takes advantage of the fact that almost all man-made sources would have a non-Gaussian distribution. See Fig. 1 taken from [9]. RFI is detected by measuring the amount of deviation of the kurtosis parameter from the value associated with normality.

The kurtosis algorithm measures higher order central moments of the incoming radiometer signal. The second central moment (variance) obtained over a particular integration period is equivalent to the system noise temperature measured by the radiometer-i.e., it is the output of a traditional radiometer, and what we know as the measured brightness temperature $T_{B}$ (after proper calibration). The ratio of the fourth central moment to the squared second central moment gives the kurtosis ratio, as shown in

$$
k=\frac{\left\langle x^{4}(t)\right\rangle}{\left\langle x^{2}(t)\right\rangle^{2}}
$$



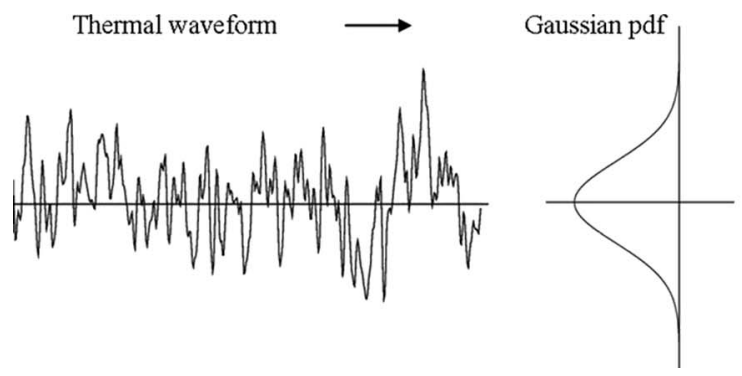

Fig. 1. Waveforms and probability density functions.
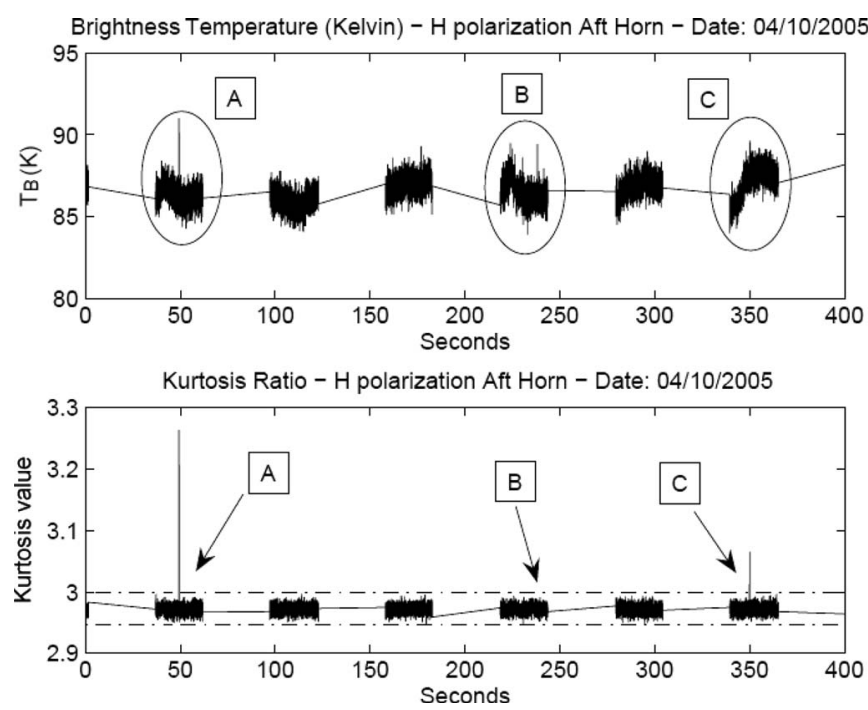

Fig. 2. $T_{B}$ and kurtosis data from the North Sea.

where $x(t)$ is the predetection signal and \langle\rangle represents statistical expectation. For natural thermal emissions, the distribution is Gaussian, and the aforementioned ratio should ideally be equal to three. If the signal is corrupted by man-made RFI, the distribution should deviate from normality, and thus, $k$ should deviate from three. A pulsed sinusoidal waveform of low duty cycle (radar-type signal) will have a kurtosis that is larger than three. A continuous sinusoidal signal $(\mathrm{CW})$ will have a kurtosis $=$ 1.5. This property can be used to flag RFI-contaminated signals. However, there is a "blind spot": A sinusoidal signal with 50\% duty cycle will also have a kurtosis $=3$.

The algorithm is insensitive to change in the second moment, and natural variations in the brightness temperature will not be falsely flagged as RFI.

Since a finite number of samples are used for calculating kurtosis, the ratio itself behaves as a random variable. Thus, kurtosis has a standard deviation associated with it (see [10]). This is expressed in

$$
\operatorname{var}(k)=\frac{24}{N}
$$

(for $N$ that is large) where $N$ is the number of statistically independent samples. This means that, when deciding whether the kurtosis value deviates from three (as an indication of RFI), a certain guard band is necessary to avoid massive false alarms. EMIRAD-2 uses \pm 4 standard deviations, corresponding to a noncontaminated signal, as guard band. This is shown in Figs. 2 and 3 .
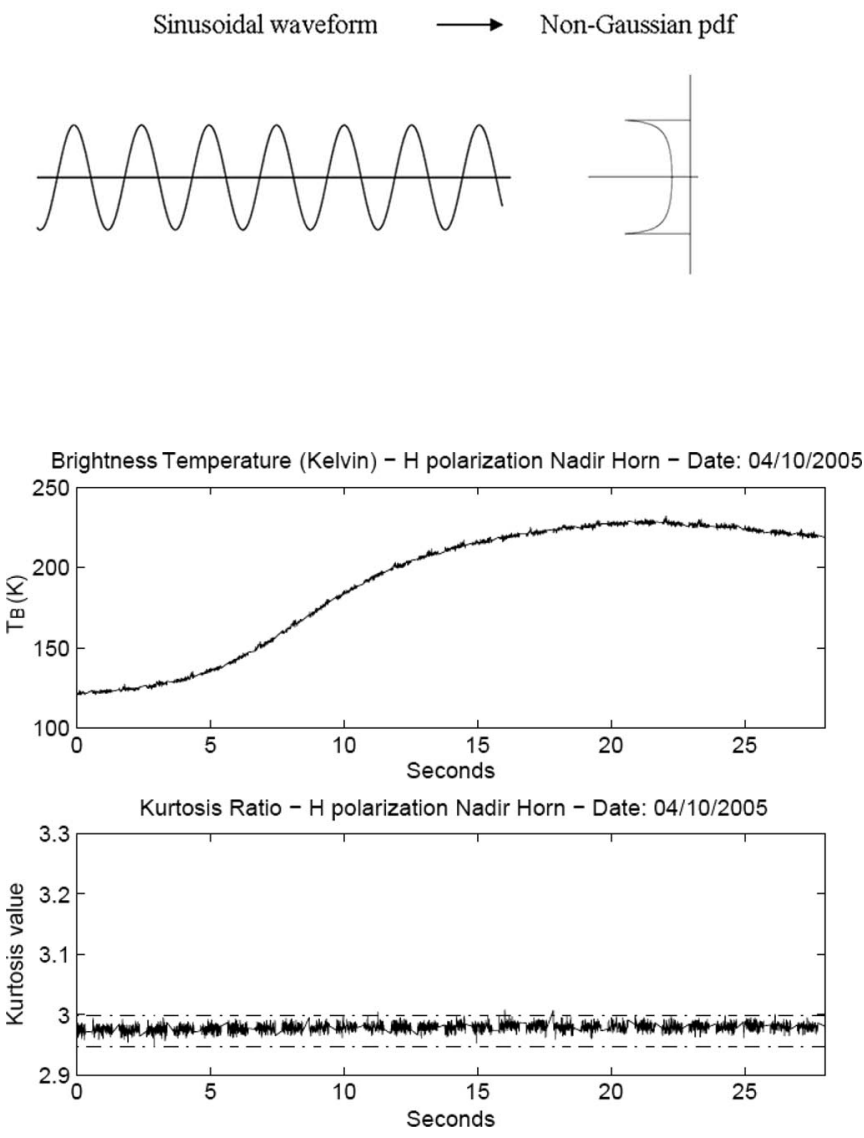

Fig. 3. Kurtosis independent from coastal crossing.

It should be noted that the kurtosis parameter itself is affected by other factors such as digitization, bit length, clipping, number of samples, correlation, filtering, nonstationarity, etc., which add a certain noise margin to it and have to be taken into account [11]. These factors cause the kurtosis ratio mean to be slightly lower than three, as seen in Figs. 2 and 3.

Theoretically (no noise and no statistical uncertainties), any deviation from three in the kurtosis parameter will be a safe RFI detector-apart from the 50\%-duty-cycle blind spot. This means that both pulsed radarlike signals (very often encountered) and CW-like signals (could be expected from some communication systems) will be safely detected. However, due to the statistical behavior of the kurtosis itself and the fact that a harmful RFI may only contribute a few kelvins to the measured $T_{B}$, the situation is less ideal. Considering a $\mathrm{CW}$ signal, the kurtosis will, in this case, not drop to 1.5 , but to somewhere between 3 and 1.5, depending on the RFI to $T_{B}$ ratio. A pulsed RFI of low duty cycle will still typically result in a quite-large kurtosis value. In effect, the already-mentioned 50\%-duty-cycle blind spot is rather a blind range. Thus, kurtosis is particularly efficient in detecting low-duty-cycle RFI. Experience has shown, however, that most RFI encountered is of this nature, so the kurtosis method can be regarded as a very efficient RFI indicator, but with its weaknesses that should not be forgotten. A special feature that should be recalled is the fact that, when duty cycle is discussed in the present context, it is the duty cycle within the time frame over which kurtosis is calculated. This means that, if the radiometer system integrates and calculates $T_{B}$ and kurtosis at a rate corresponding to, for example, $8 \mathrm{~ms}$, 
an RFI pulse that is longer than $4 \mathrm{~ms}$ can be placed in time such that one 8 -ms sample sees a 50\%-duty-cycle signal that will be undetected, even if it is of a considerable and harmful magnitude.

One thing is detection of RFI, and another is possible mitigation. In general, samples that have been flagged as RFI contaminated must be discarded. As already stated, RFI is often of pulsed nature. Thus, it is of great importance to operate the radiometer system with short integration time and fast data rate such that efficient mitigation can be carried out without too severe loss of data. The EMIRAD-2 instrument operates with integration times (and corresponding recording rates) in the millisecond range for this reason (microsecond rate for special purposes).

\section{EMIRAD-2 POLARIMERIC RADIOMETER SYSTEM}

The EMIRAD-2 radiometer system is developed and operated by the Technical University of Denmark. The system is installed in a Short SC-7 Skyvan aircraft owned and operated by Helsinki University of Technology (during the Australian campaign, the instrument was, however, mounted on a Rockwell Aero Commander). The radiometer system is a fully polarimetric (all four Stokes parameters) digital system with subharmonic sampling. The radiometer system comprises several units: antennas, the radiometer, data recording and control units, attitude and position sensors.

The antenna system consists of two large Potter horns and waveguide orthomode transducers for almost ideal antenna patterns and low loss. The Potter horns have side- and backlobes being suppressed by more than $40 \mathrm{~dB}$, while the crosspolarization level is even smaller. One antenna having a $38^{\circ}$ half-power beamwidth (HPBW) is pointed nadir, the other $\left(31^{\circ}\right.$ HPBW) is pointed $40^{\circ}$ aft. Thus, a point on the ground is covered twice with different incidence angle and very short time interval. The signals from the two antennas are multiplexed through one polarimetric radiometer receiver-fast enough to get full ground coverage at both channels, taking the footprint size and aircraft speed into consideration. It must be realized that, if an RFI spike is detected in, for example, the nadir antenna signal, its response in the aft antenna signal cannot be examined due to the multiplexing.

The receiver is a fully polarimetric correlation radiometer with direct sampling. Two fast A/D converters directly sample the L-band signals. The sampling frequency is $139.4 \mathrm{MHz}$. The data from the two A/D converters are fed into a fieldprogrammable gate array where detection and correlation takes place, as well as integration to $8 \mathrm{~ms}$ (from 2008: $1 \mathrm{~ms}$ ) and output to storage ("slow data" for normal users), and also only integrated to $1.8 \mu$ s (from 2008: $14.7 \mu \mathrm{s}$ ) before output to storage ("fast data" for special investigations). Moreover, kurtosis is calculated for the same two time intervals and output to storage. In the "slow" case, the number of samples in the calculations is very large, resulting in a very clean kurtosis with low variance according to (2).

The radiometer is designed for optimum radiometric sensitivity, so the full 27-MHz-wide protected band is used. Thus, external radiation that is very close to the protected band might enter the radiometer, but based on experience from campaigns over the North Sea and Denmark with a previous instrument of similar design, where RFI was found not to be a problem, the tradeoff between RFI susceptibility and radiometric sensitivity favored the latter. In the new design, RFI is mitigated using the kurtosis method. The relatively moderate percentages of data samples, which had to be discarded under normal campaign conditions, is an indication of the usefulness and validity of this design. It has, however, to be recalled that, whenever RFI is detected in the system, it is impossible to say whether it is RFI within the protected band or strong active signals just outside the band.

The measured sensitivities for 8-ms integration time are as follows: $0.9 \mathrm{~K}$ for the $\mathrm{H}$ (horizontally polarized) and $\mathrm{V}$ (vertically polarized) channels and $1.2 \mathrm{~K}$ for the third and fourth Stokes parameters.

In summary, the instrument specifications are the following.

1) Correlation radiometer with direct sampling.

2) Fully polarimetric (i.e., four Stokes).

3) Frequencies: 1400-1427 MHz (-1-dB bandwidth), 1399-1428 MHz (-3-dB bandwidth), and 1390$1436 \mathrm{MHz}$ ( $-60-\mathrm{dB}$ bandwidth).

4) Digital radiometer with $139.4-\mathrm{MHz}$ sampling.

5) "Slow data" integrated to $8 \mathrm{~ms}$ (from 2008: $1 \mathrm{~ms}$ ) recorded on $\mathrm{PC}$.

6) "Fast data" integrated to $1.8 \mu \mathrm{s}$ (from 2008: $14.7 \mu \mathrm{s}$ ) recorded on hard disk.

7) Sensitivity: $0.1 \mathrm{~K}$ for $1-\mathrm{s}$ integration time.

8) Stability: better than $0.1 \mathrm{~K}$ over $15 \mathrm{~min}$.

9) Calibration: internal loads and noise diode.

10) Two antennas-one pointing nadir and one pointing $40^{\circ}$ aft.

11) Antennas are Potter horns with $38^{\circ}$ (nadir) and $31^{\circ}$ (aft) HPBW.

12) Footprint around $700 \mathrm{~m}$ per 1000 -m flight altitude.

See [12] for further information about the EMIRAD-2 system.

\section{EXPERIENCE From the Airborne COSMOS CAMPAIGNS}

In this section, examples of data from the CoSMOS campaigns will be presented and discussed. The data were acquired with the EMIRAD-2 radiometer system.

The CoSMOS campaigns have covered a range of targets at very different locations. In the fall of 2005, agricultural areas were covered in Australia. In the spring of 2006, the North Sea west of Norway was the target [13]. Finland was the target area in 2007 with campaigns over the ice-covered sea in winter and over the open sea in summer. Finally, 2008 saw the coverage of agricultural and urban areas in Germany and Spain, as well as transits including France and the Mediterranean Sea.

\section{A. Examples of Signals}

Consider a typical flight over the North Sea. The aircraft operates out of Stavanger, transits to an area almost midway between Norway and Great Britain, carries out a number of circles in order to investigate possible azimuth signatures of the wind-driven sea, and returns to Stavanger.

Fig. 2 shows 8 -ms brightness temperature $\left(T_{B}\right)$ data from the transit of such a flight. The radiometric data are not continuous as the two antenna horns (nadir and $40^{\circ}$ aft looking) are multiplexed through the radiometer and, in this case, with slow rate 

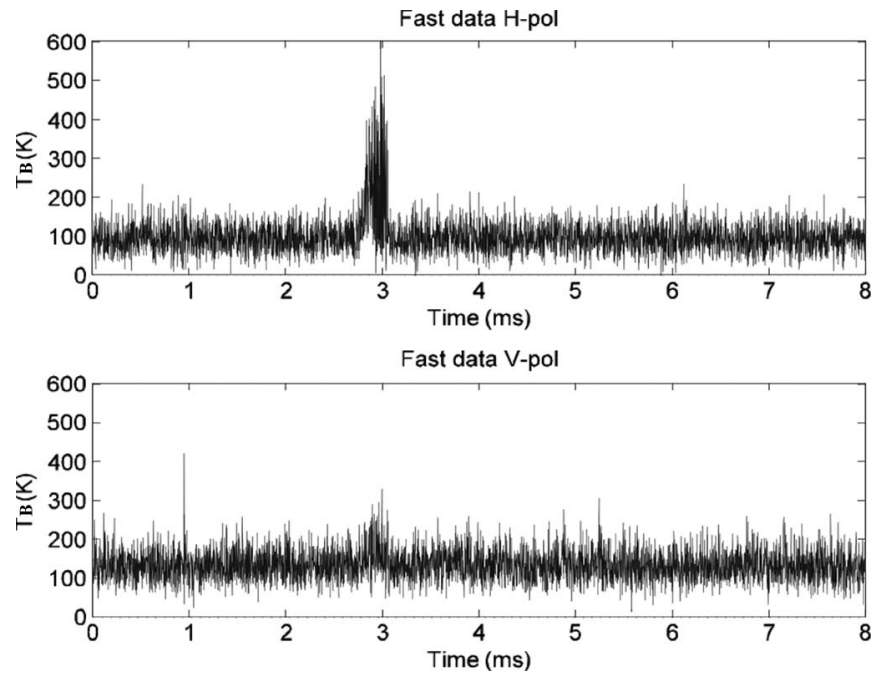

Fig. 4. Fast data example A ( $\mathrm{H}$ and $\mathrm{V}$ pol).

to save on the switching events (mechanical low-loss switch) during the many long transits of the North Sea campaign.

The figure shows $400 \mathrm{~s}$ of slow (8-ms) data measured by the aft horn, with the top plot indicating $T_{B}$ (in kelvins) and the bottom plot indicating the kurtosis. The dashed lines in the bottom plot indicate the kurtosis thresholds within which data are considered to be RFI free. The thresholds are based on the noise margin of the kurtosis statistics. The kurtosis mean is slightly less than three, as explained in Section II. Cases A and $\mathrm{C}$ shown in the plots are flagged as RFI. By comparing them, it is noticed from the top plot that there is a clear spike in the brightness data for the first case (A), whereas there is negligible difference in the value of the second case (C) compared to its surroundings. This demonstrates the ability of the kurtosis algorithm to detect man-made interference near the noise margin of the radiometer data. Case B is a counterexample, where the $T_{B}$ plot clearly indicates a spike, and this might cause the data sample to be falsely flagged as RFI if some kind of threshold algorithm was used. On the other hand, the bottom plot indicates the kurtosis completely within the set noise margins, thus demonstrating that the sample is a part of a natural thermal emission. An alternative explanation of case B might be that there is indeed RFI, but not detected by kurtosis-which definitely is a possibility: A 50\%-duty-cycle signal (understood as 50\% within the 8-ms time frame), for example, will have kurtosis $=3$ and will thus not be detected.

Each slow data sample integrated over a period of $8 \mathrm{~ms}$ has approximately 4500 corresponding fast data samples, see Figs. 4 and 5. If any slow data sample is flagged as RFI by kurtosis, by observing the associated fast data samples, it is possible to determine the nature of the RFI (pulses, CW-like) and, in the typical case of pulses, to measure the exact amount of RFI contribution to the measured temperature value. The integrated value is calculated before and after discarding all the obvious RFI spikes. It is also seen that such fast sampling offers an alternative to kurtosis flagging of RFI as the RFI spikes are generally very visible.

As already stated, the kurtosis algorithm is insensitive to changes in the brightness temperature, as measured by the antenna. Even the large and fast variations encountered when
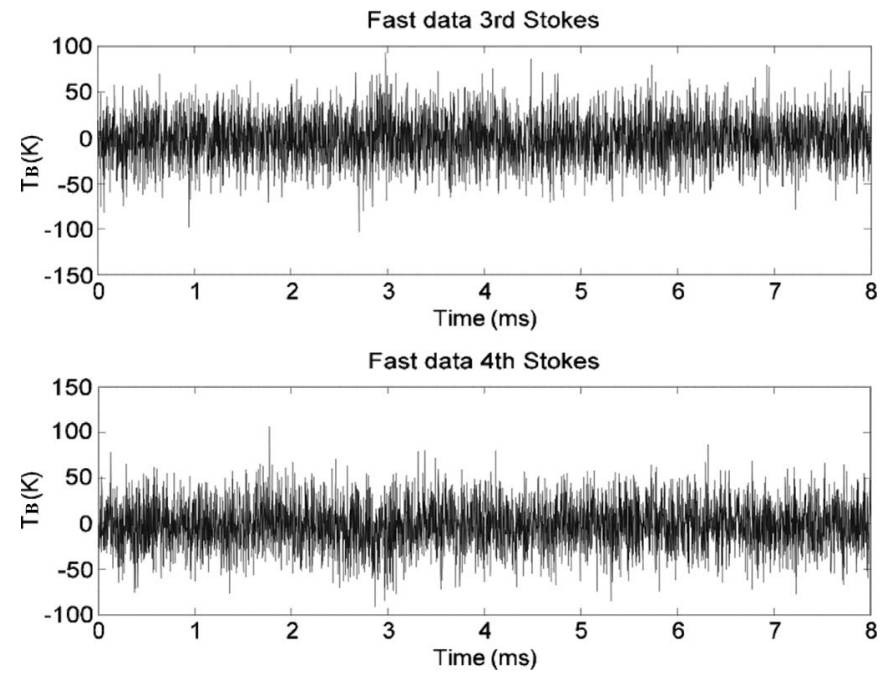

Fig. 5. Fast data example A (third and fourth Stokes).

TABLE I

RFI PERCENTAGES (Australia)

\begin{tabular}{|c|c|c|c|c|c|}
\hline Date & Aft $\mathrm{H}$ & Aft V & Nadir $\mathrm{H}$ & Nadir V & Flight duration \\
\hline $15 / 11-05$ & $2.99 \%$ & $0.83 \%$ & $15.0 \%$ & $2.58 \%$ & $2 \mathrm{~h} 25 \mathrm{~m}$ \\
\hline $27 / 11-05$ & $2.26 \%$ & $0.55 \%$ & $17.4 \%$ & $2.69 \%$ & $1 \mathrm{~h} 24 \mathrm{~m}$ \\
\hline $29 / 11-05$ & $2.94 \%$ & $0.78 \%$ & $16.0 \%$ & $1.22 \%$ & $1 \mathrm{~h} 27 \mathrm{~m}$ \\
\hline $3 / 12-05$ & $5.44 \%$ & $5.70 \%$ & $30.0 \%$ & $5.34 \%$ & $1 \mathrm{~h} 26 \mathrm{~m}$ \\
\hline $6 / 12-05$ & $4.03 \%$ & $2.10 \%$ & $21.8 \%$ & $4.97 \%$ & $1 \mathrm{~h} 29 \mathrm{~m}$ \\
\hline
\end{tabular}

crossing a coastline will not result in false alarms. Fig. 3 shows an experimental verification of this theoretical fact.

It has also been attempted to move an absorber in and out of the antenna beam just in front of the aperture, while the antenna was pointing toward the sky via an aluminum reflector on the apron behind the aircraft. Even this unrealistically fast change in brightness temperature did not trigger the kurtosis flag.

Experience shows that RFI can take many forms. Fig. 2 shows a rather typical situation with low RFI. Extremes with much more severe RFI have been experienced not only near cities/airports (rather often) but also even on a few occasions in the middle of the North Sea.

\section{B. Percentages Flagged}

The "slow" data (8 ms until and through 2007 and $1 \mathrm{~ms}$ in 2008) from all CoSMOS campaigns have been processed and flagged using the kurtosis method. Tables I-VI show the percentage of data being flagged concerning selected missions. Percentages are shown individually for the two horns and polarizations. The footprint is typically between $700 \mathrm{~m}$ (over the target areas) and $2000 \mathrm{~m}$ (transit).

There are two striking features in Table II: First, it is seen that, in general, the percentages over sea are very modest, and if all flagged data are discarded before entering into analysis, the RFI influence is mitigated in a simple way, and the influence on radiometric $\Delta T$ is totally insignificant. Second, the missions on April 22 and 29 are very special. When looking at the data, it is found that, for a substantial period, while flying midway between Norway and England, there is almost 100\% RFI of pulsed nature. The source is unknown, but it is believed to be 
TABLE II

RFI PERCENTAGES (NORTH SEA)

\begin{tabular}{|c|c|c|c|c|c|}
\hline Date & Aft H & Aft V & Nadir $\mathrm{H}$ & Nadir V & Flight duration \\
\hline $6 / 4-06$ & $0.44 \%$ & $0.53 \%$ & $0.31 \%$ & $0.49 \%$ & $3 \mathrm{~h} 58 \mathrm{~m}$ \\
\hline $9 / 4-06$ & $0.06 \%$ & $0.37 \%$ & $0.06 \%$ & $0.44 \%$ & $2 \mathrm{~h} 51 \mathrm{~m}$ \\
\hline $10 / 4-06$ & $0.03 \%$ & $0.36 \%$ & $0.05 \%$ & $0.43 \%$ & $2 \mathrm{~h} 57 \mathrm{~m}$ \\
\hline $12 / 4-06$ & $0.04 \%$ & $0.31 \%$ & $0.04 \%$ & $0.36 \%$ & $3 \mathrm{~h} 04 \mathrm{~m}$ \\
\hline $13 / 4-06$ & $0.57 \%$ & $1.02 \%$ & $0.87 \%$ & $1.16 \%$ & $2 \mathrm{~h} 54 \mathrm{~m}$ \\
\hline $15 / 4-06$ & $0.05 \%$ & $0.61 \%$ & $0.06 \%$ & $0.72 \%$ & $3 \mathrm{~h} 24 \mathrm{~m}$ \\
\hline $16 / 4-06$ & $1.97 \%$ & $2.44 \%$ & $3.37 \%$ & $1.52 \%$ & $2 \mathrm{~h} 28 \mathrm{~m}$ \\
\hline $18 / 4-06$ & $0.13 \%$ & $0.46 \%$ & $0.06 \%$ & $0.56 \%$ & $3 \mathrm{~h} 10 \mathrm{~m}$ \\
\hline $19 / 4-06$ & $0.70 \%$ & $1.45 \%$ & $2.34 \%$ & $0.90 \%$ & $3 \mathrm{~h} 15 \mathrm{~m}$ \\
\hline $22 / 4-06$ & $35.9 \%$ & $41.6 \%$ & $43.9 \%$ & $18.0 \%$ & $3 \mathrm{~h} 01 \mathrm{~m}$ \\
\hline $25 / 4-06$ & $0.05 \%$ & $0.27 \%$ & $0.06 \%$ & $0.29 \%$ & $2 \mathrm{~h} 48 \mathrm{~m}$ \\
\hline $29 / 4-06$ & $31.2 \%$ & $35.4 \%$ & $53.3 \%$ & $18.7 \%$ & $2 \mathrm{~h} 57 \mathrm{~m}$ \\
\hline $30 / 4-06$ & $0.99 \%$ & $0.28 \%$ & $0.44 \%$ & $0.60 \%$ & $1 \mathrm{~h} 08 \mathrm{~m}$ \\
\hline
\end{tabular}

TABLE III

RFI Percentages (Finland)

\begin{tabular}{|c|c|c|c|c|c|}
\hline Date & Aft $\mathrm{H}$ & Aft V & Nadir H & Nadir V & Flight duration \\
\hline $8 / 3-07$ & $0.86 \%$ & $1.6 \%$ & $0.59 \%$ & $0.81 \%$ & $1 \mathrm{~h} 22 \mathrm{~m}$ \\
\hline $11 / 3-07$ & $0.07 \%$ & $0.16 \%$ & $0.12 \%$ & $0.10 \%$ & $3 \mathrm{~h} 03 \mathrm{~m}$ \\
\hline $12 / 3-07$ & $10.8 \%$ & $21.5 \%$ & $8.6 \%$ & $20.8 \%$ & $2 \mathrm{~h} 27 \mathrm{~m}$ \\
\hline $13 / 3-07$ & $0.17 \%$ & $0.22 \%$ & $0.12 \%$ & $0.09 \%$ & $2 \mathrm{~h} 25 \mathrm{~m}$ \\
\hline $13 / 8-07$ & $0.08 \%$ & $0.50 \%$ & $0.07 \%$ & $0.56 \%$ & $3 \mathrm{~h} 13 \mathrm{~m}$ \\
\hline $13 / 8-07$ & $0.23 \%$ & $3.8 \%$ & $0.61 \%$ & $0.46 \%$ & $1 \mathrm{~h} 46 \mathrm{~m}$ \\
\hline $15 / 8-07$ & $0.22 \%$ & $0.42 \%$ & $0.11 \%$ & $0.38 \%$ & $3 \mathrm{~h} 17 \mathrm{~m}$ \\
\hline
\end{tabular}

TABLE IV

RFI PERCENTAGES (MÜNCHEN)

\begin{tabular}{|c|c|c|c|c|c|}
\hline Date & Aft $\mathrm{H}$ & Aft V & Nadir H & Nadir V & Flight duration \\
\hline $8 / 4-08$ & $2.74 \%$ & $2.80 \%$ & $1.79 \%$ & $2.70 \%$ & $1 \mathrm{~h} 46 \mathrm{~m}$ \\
\hline $14 / 4-08$ & $1.66 \%$ & $1.82 \%$ & $1.70 \%$ & $1.89 \%$ & $1 \mathrm{~h} 56 \mathrm{~m}$ \\
\hline $18 / 4-08$ & $4.16 \%$ & $3.57 \%$ & $3.46 \%$ & $3.51 \%$ & $2 \mathrm{~h} 00 \mathrm{~m}$ \\
\hline
\end{tabular}

TABLE V

RFI PeRCENTAges (VALENCIA)

\begin{tabular}{|c|c|c|c|c|c|}
\hline Date & Aft H & Aft V & Nadir H & Nadir V & Flight duration \\
\hline $22 / 4-08$ & $3.28 \%$ & $3.35 \%$ & $1.82 \%$ & $1.88 \%$ & $1 \mathrm{~h} 27 \mathrm{~m}$ \\
\hline $24 / 4-08$ & $8.93 \%$ & $4.64 \%$ & $3.34 \%$ & $2.59 \%$ & $1 \mathrm{~h} 30 \mathrm{~m}$ \\
\hline $28 / 4-08$ & $4.50 \%$ & $2.87 \%$ & $2.39 \%$ & $2.31 \%$ & $1 \mathrm{~h} 40 \mathrm{~m}$ \\
\hline $2 / 5-08$ & $34.6 \%$ & $30.5 \%$ & $38.5 \%$ & $32.9 \%$ & $1 \mathrm{~h} 30 \mathrm{~m}$ \\
\hline
\end{tabular}

TABLE VI

RFI PERCENTAges (TRANSit)

\begin{tabular}{|c|c|c|c|c|c|}
\hline Where? & $\mathrm{Aft} H$ & Aft V & Nadir H & Nadir V & Flight duration \\
\hline Valencia-Casablanca & $42.4 \%$ & $18.1 \%$ & $10.7 \%$ & $23.3 \%$ & $45 \mathrm{~m}$ \\
\hline Casablanca - Marseille & $33.2 \%$ & $24.3 \%$ & $20.63 \%$ & $28.32 \%$ & $1 \mathrm{~h} 40 \mathrm{~m}$ \\
\hline Marseille - Dole & $14.1 \%$ & $17.7 \%$ & $10.7 \%$ & $12.9 \%$ & $2 \mathrm{~h} 09 \mathrm{~m}$ \\
\hline Dole - Luxembourg & $7.09 \%$ & $5.67 \%$ & $4.20 \%$ & $4.53 \%$ & $1 \mathrm{~h} 29 \mathrm{~m}$ \\
\hline Luxembourg - Wüstebach & $0.11 \%$ & $0.19 \%$ & $0.04 \%$ & $0.17 \%$ & $2 \mathrm{~m}$ \\
\hline Wüstebach - Paderborn & $3.83 \%$ & $4.89 \%$ & $3.23 \%$ & $4.15 \%$ & $40 \mathrm{~m}$ \\
\hline Paderborn - Kalmar & $3.47 \%$ & $2.63 \%$ & $1.57 \%$ & $1.75 \%$ & $2 \mathrm{~h} 31 \mathrm{~m}$ \\
\hline Kalmar - Helsinki & $0.57 \%$ & $0.73 \%$ & $0.32 \%$ & $0.42 \%$ & $2 \mathrm{~h} 23 \mathrm{~m}$ \\
\hline
\end{tabular}

external, since the aircraft and the installation were completely unchanged for the many North Sea sorties.

On April 16, a much smaller yet significant increase in RFI is noted. This and the cases discussed earlier illustrate the experience that RFI might well change significantly from day to day over the same area.

Concerning the Australian data set (see Table I), the percentages are somewhat higher but still manageable in most cases. It is noted that the RFI level is consistently higher in the nadir $\mathrm{H}$ channel. It is difficult to imagine this being a result of external sources, bearing in mind the complicated flight patterns used for covering a scientific land site (the RFI should move from $\mathrm{H}$ to $\mathrm{V}$ and from the nadir channel to the aft channel). Interference from some equipment in the aircraft cannot be excluded. A signal that is too small to be noticed by the operator in real time yet large enough to be detected by kurtosis could be responsible (kurtosis was calculated offline). The effect was only seen in the Australian data where a local airplane was used-not when the Skyvan was used for the larger parts of the CoSMOS campaign.

In 2007, two campaigns were carried out in Finland: one campaign in northern Finland, and another one near Helsinki. The percentages flagged concerning these two campaigns are shown in Table III. It is seen that the percentages are generally very modest apart from a special case on $12 / 3$. The cause is not known, but the aircraft can hardly be suspected as being the source, since nothing was changed from day to day. Thus, the case again illustrates that significant daily variations can be observed. It is clear that RFI was not a major problem during these two campaigns.

In 2008, the so-called Rehearsal Campaign was carried out, essentially covering land sites in Germany and Spain. The flights also included transits over the northwestern Mediterranean Sea, as well as a transit over France going all the way from south to north. The percentages flagged for this campaign are seen in Tables IV-VI.

The RFI conditions around München are benign and generally also around Valencia, apart from the 2/5 sortie (reason not known, see comment to Table III), whereas it is serious during the first part of the transit flight from Valencia to Helsinki.

It is observed that the percentages of data flagged for RFI show some regional variability. Generally, very small amounts are flagged over the North Sea and in Finland. Also, near München and Valencia, small values are noted. Australia has relatively more data being flagged, but still, a manageable level is experienced. High levels are encountered in southern France-or perhaps, one could state "both sides of the French/ Spanish border." It is illustrative to observe how the percentage gradually decreases to very small values as the transit progresses toward north.

\section{Histograms}

Fig. 6 shows histograms of the amount of data affected by RFI for all days of the CoSMOS North Sea campaign, with the exception of April 22 and 29, which are regarded as very nontypical (particularly amid open ocean), when integrated over a period of $1 \mathrm{~s}$. The plot shows how the flagged data are distributed according to the RFI contribution to $T_{B}$. The calculations are carried out using the fast data, as described in 

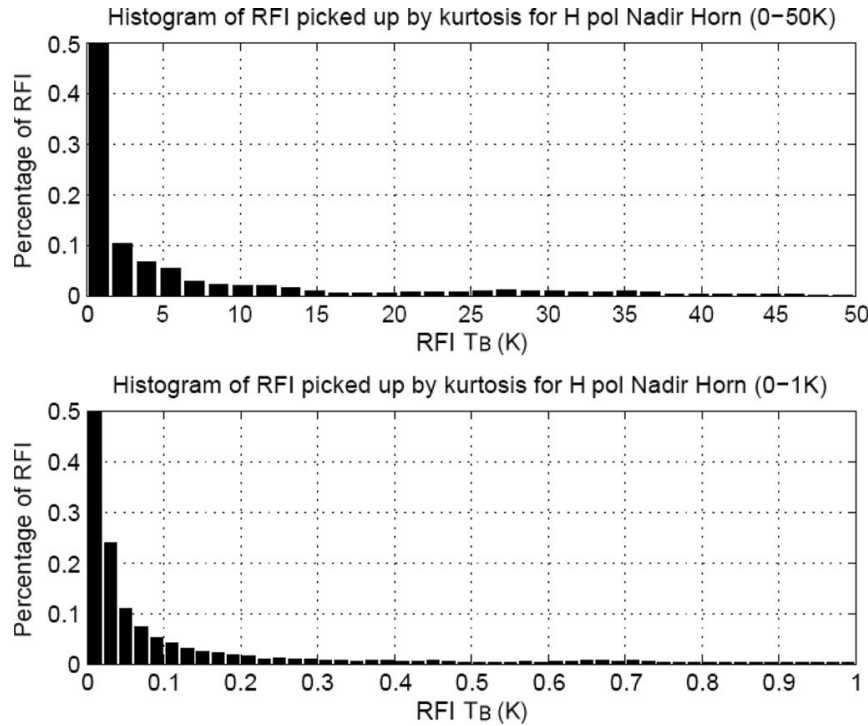

Fig. 6. Impact of RFI in terms of $T_{B}$. North Sea data. Note that the first bar in both cases is truncated in order to obtain a reasonable scaling. The first bar in the top plot is 1.94, and it is 0.94 in the bottom plot.
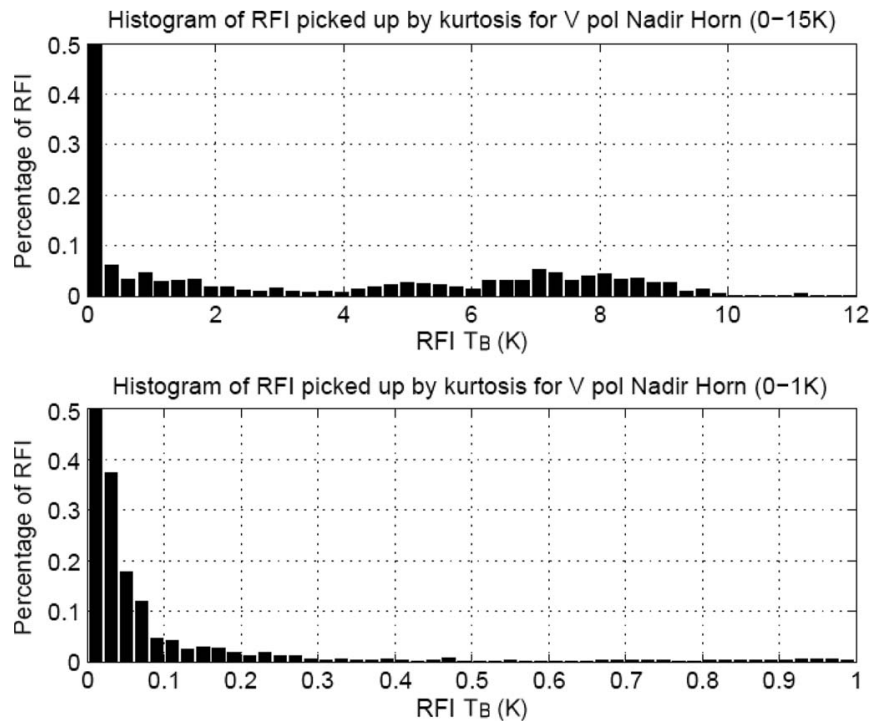

Fig. 7. Impact of RFI in terms of $T_{B}$. Australia data. Note that the first bar in both cases is truncated in order to obtain a reasonable scaling. The first bar in the top plot is 1.9 , and it is 0.97 in the bottom plot.

Section IV-A. The integrated $T_{B}$ value is calculated before and after discarding all the obvious RFI spikes.

About half of the RFI flagged by the kurtosis algorithm is below the 1-K region, and a significant part is actually below $0.1 \mathrm{~K}$, which can be ignored, even for ocean applications. A significant influence of RFI is observed around the 2-15-K region, as indicated by the top plot. Such RFI is very difficult to eliminate using conventional threshold algorithms. Finally, there are a few cases between 15 and $50 \mathrm{~K}$ that will show up clearly in the brightness temperature data and can be eliminated using classical methods.

Fig. 7 shows histograms of the amount of data affected by RFI for all days during the CoSMOS Australia campaign. Again, the plot shows how the flagged data are distributed according to the RFI contribution to $T_{B}$.

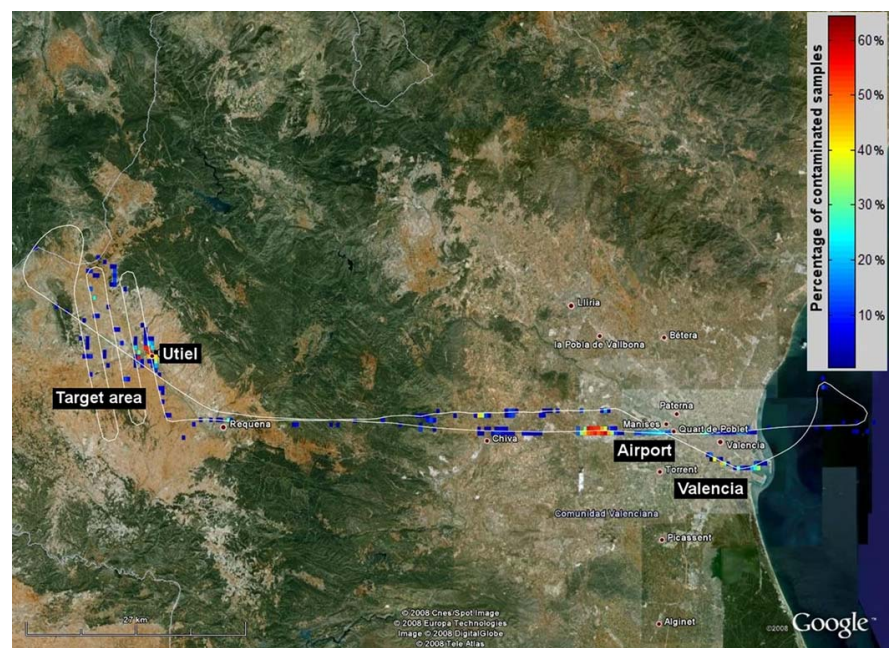

Fig. 8. Valencia-overview.

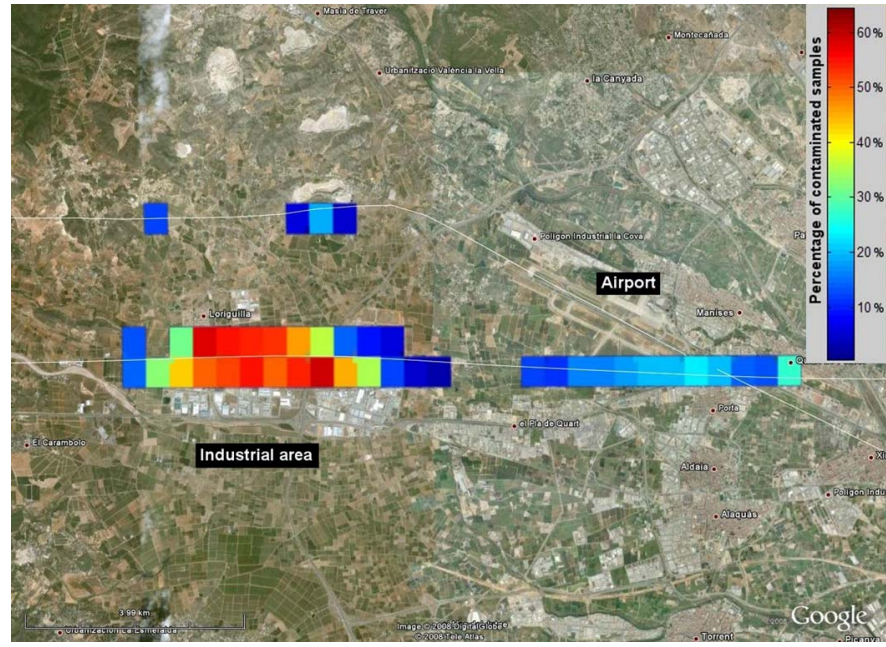

Fig. 9. Valencia-airport and industrial zone.

Even though CoSMOS Australia has more RFI than the campaigns in the North Sea, the intensity of RFI is relatively less. Fig. 6 clearly shows that RFI contributions can go up to $50 \mathrm{~K}$ during the Norway campaign, whereas for Australia, Fig. 7 shows that it hardly went above $10 \mathrm{~K}$. There appears to be a bump around 6-10 K, which, as discussed earlier, is hard for normal threshold algorithms to detect.

From the histograms, it is clear that a large part of the flagged data have RFI contribution below the 1-K level, which might be ignored for soil-moisture purposes.

In summary, it can be stated that the kurtosis algorithm flags harmful RFI, but in addition, a significant amount of weak and nonharmful RFI is also flagged. Experience indicates that up to $50 \%$ of the flagged data would not have contributed to the brightness temperature data with a harmful amount (application dependent: contributions below $1-\mathrm{K}$ might well be acceptable for soil-moisture sensing).

\section{Location of RFI}

It is of interest to investigate where RFI is typically located. An example shown in Figs. 8-10 is the flight on April 22 near Valencia, which was part of the 2008 Rehearsal Campaign. 


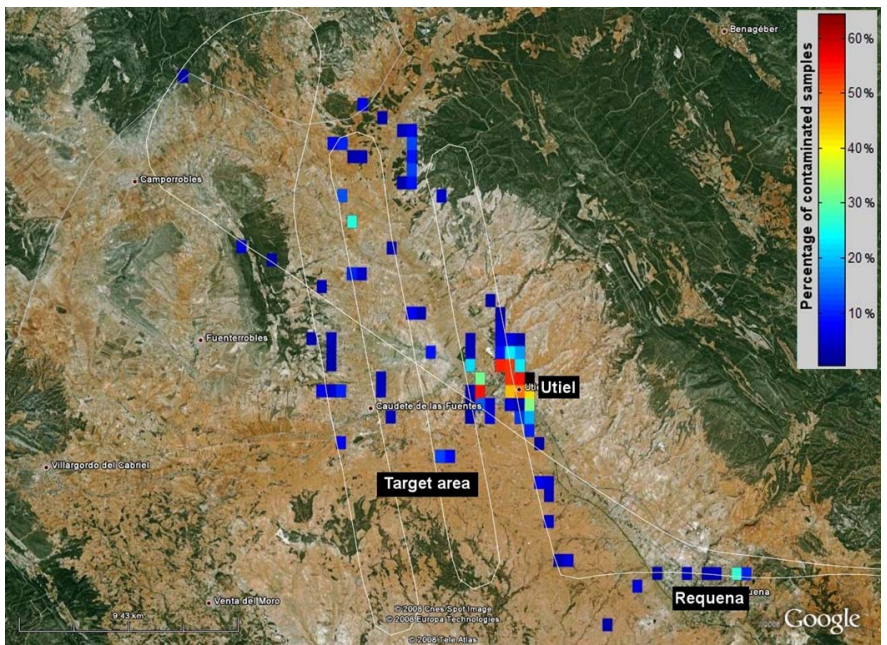

Fig. 10. Valencia—campaign area.

The RFI percentages are color coded according to the scale shown on the right-hand side of the images. An RFI pixel is about $550 \times 550 \mathrm{~m}$ and corresponds to approximately 4500 1 -ms samples.

Fig. 8 shows an overview of the Valencia flight, starting from the city's airport, transit to the campaign area, coverage of this area, transit to the open sea for calibration check, and finally returning to the airport. Fig. 9 zooms in on the area around the airport and an industrial zone, while Fig. 10 concentrates on the campaign area.

The RFI appears to be of a rather spotty nature, mostly concentrated around the airport, the industrial zone, and near town Utiel in the campaign area. It is, however, also noted that some RFI is detected here and there throughout the campaign area, and this has to be accounted for. Similar results were found for most days in the Valencia area.

Maps were also generated for the München area, and the situation is generally the same: very little RFI in the rural campaign area, and some hot spots near airports and cities.

Note, however, that, when RFI is detected at a certain time, hence at a certain aircraft position, it is in the figures indicated at the corresponding antenna footprint position. This does not necessarily mean that the emitter of the disturbing signal has that geographical position: The radiometer antenna has a certain directivity pattern, and a strong signal far away from the antenna boresight might enter the radiometer system. However, the fact that certain areas (airports, industrial zones, and special installations) consistently appear as RFI contaminated on different flights indicate that, in general, the maps can be regarded as maps of disturbing sources.

Concerning the transit from Valencia to Helsinki, the situation is generally as before-only noting that RFI levels are much higher until well up into France: Disturbances are predominantly around cities and less over rural areas.

The very large CoSMOS experimental data set confirms the expectations that, over land areas, the RFI is much concentrated near airports, industrial zones, and urban areas in general, while very little RFI is detected in rural areas. For airborne (and ground-based) campaigns, this is good news as the target areas are generally in rural areas.
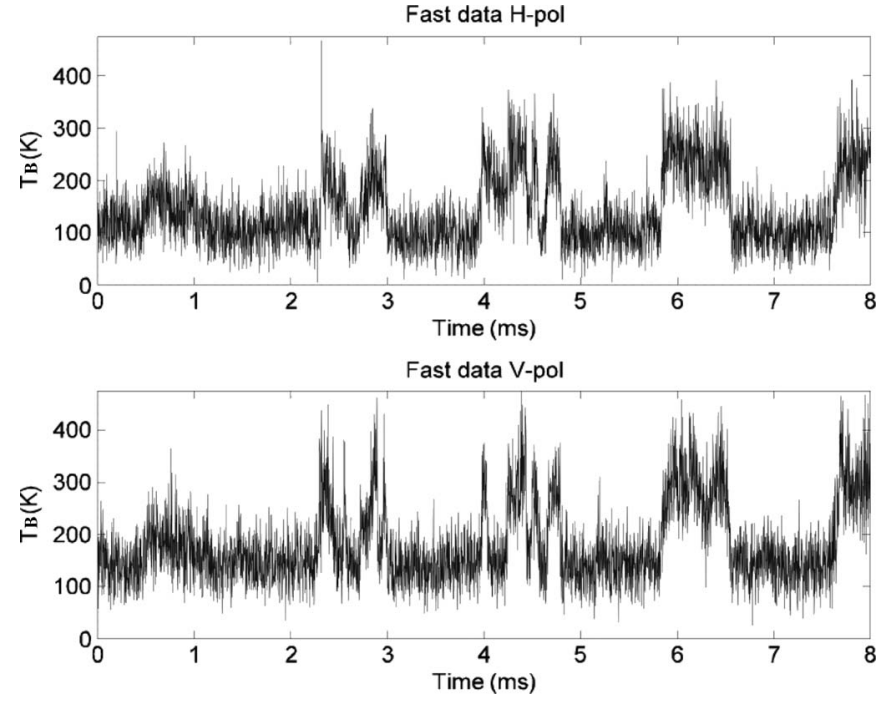

Fig. 11. Fast data example B ( $\mathrm{H}$ and $\mathrm{V}$ pol).

\section{E. Two Fast Data Examples}

In the following will be discussed two examples of RFI flagged 8 -ms samples, using the fast data $(1.8 \mu \mathrm{s})$, to show typical signal details, the potential of fast data for detection and mitigation of pulselike signals, and, finally, the potential of fully polarimetric data.

In Figs. 4 and 5, one relatively strong pulse contributes $2.5 \mathrm{~K}$ to the $\mathrm{H}$-pol 8-ms sample and very little $(0.1 \mathrm{~K})$ to the V-pol sample, and it is invisible in the third and fourth Stokes channels.

In Figs. 11 and 12, several pulses contribute significantly to both $\mathrm{H}$ and $\mathrm{V}$ channels ( 3.6 and $7.5 \mathrm{~K}$ ) and nothing to the third Stokes channel, but a significant signal is seen in the fourth Stokes channel.

In both cases, mitigation of the RFI is quite possible: The pulses are detected by traditional amplitude methods and discarded.

In summary, RFI comes in very different ways. If very fast sampled data are available, pulsed radarlike (which is typical) RFI is quite easily detected, and kurtosis might not be needed (although a safe and convenient method). Finally, fast data are a requirement if effective mitigation is to be carried out without a substantial loss of data. Unfortunately, fast data rates strain bandwidth and/or memory resources in the data recording system.

\section{F. Using Third and Fourth Stokes Parameters to Detect RFI?}

The third and, in particular, the fourth Stokes parameter over natural targets are expected to be small at L-band. Both models and experiences with airborne and ground-based polarimetric radiometers indicate this. It seems very likely that, in general, linearly polarized RFI sources will not be aligned with the V and $\mathrm{H}$ polarizations in the Earth reference frame. This means that there will be a signal in the third Stokes parameter. Many L-band surveillance radars use circular polarization. RFI from such a radar will show up in the fourth Stokes parameter. Example B (Figs. 11 and 12) must be an example of this. Due to the reasons just mentioned, it is interesting to inspect the third 

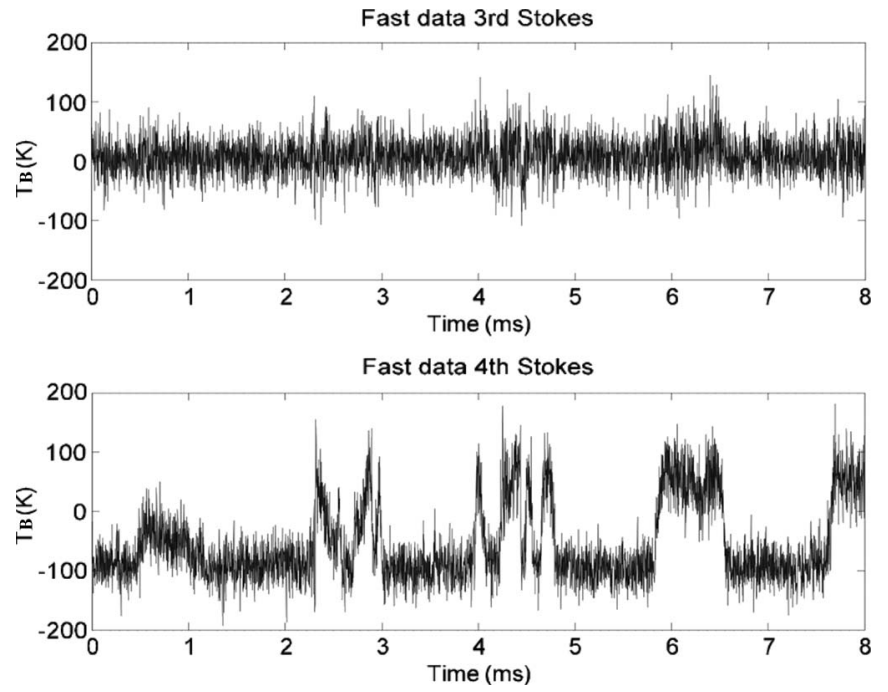

Fig. 12. Fast data example B (third and fourth Stokes).

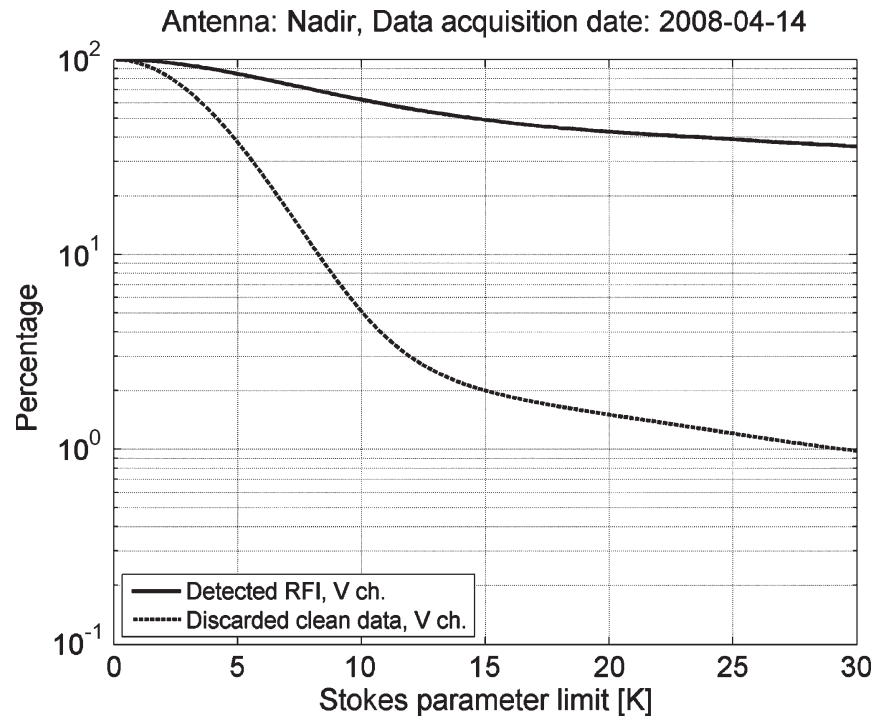

Fig. 13. Third and fourth Stokes used as RFI indicators. Detected RFI versus false alarm.

and fourth Stokes channels and maybe use a signal here as an indication of RFI.

Example B discussed earlier supports this idea, but example A does not.

In order to carry out a more general evaluation of the possibilities for detecting genuine RFI, as well as the risks of falsely flagging clean data samples as RFI-contaminated ones, a data set from the München campaign was investigated (see Fig. 13). In this analysis, it is assumed that the kurtosis method is the "truth"-i.e., when kurtosis flags a data sample, RFI is present, and no kurtosis flag means no RFI. Fig. 13 shows V-pol data, and H-pol data were also examined and plotted with almost identical curves as a result. It is assumed that the value of the third and fourth Stokes parameters over natural targets without RFI contamination is zero; hence, if they depart from that value by a prescribed amount, a sample is flagged as RFI contaminated. This amount is the horizontal axis in Fig. 13.

The vertical axis shows how many of the kurtosis-flagged data are found when the Stokes parameters are examined (upper solid curve). Also shown is the number of samples discarded (lower dotted curve), which were not flagged by kurtosis. If, for example, it is decided to discard samples where either the third or fourth Stokes parameter is greater than $10 \mathrm{~K}, 60 \%$ of the kurtosis-flagged data are detected, but in the process, also $5 \%$ of the clean data are discarded. Sixty percent might be considered as a disappointingly low number, but recall that the kurtosis algorithm flags even minor RFI, and maybe, up to half of the flagged RFI represents cases where the RFI contribution to the signal is so low that it is, in fact, not harmful and also not detected in the present analysis.

Fig. 13 shows that a maximum acceptable value in the third and fourth Stokes parameters around $10 \mathrm{~K}$, as also used in the aforementioned example, is a fair choice: The curve showing the discarded clean data drop rapidly up to about $10 \mathrm{~K}$ but then flattens out.

The present example indicates that using the third and fourth Stokes parameters as RFI indicators seems to offer an alternative or supplement to the kurtosis method, but it must be recalled that the parameters are measurement geometry dependent and that some large RFI sources might produce small Stokes parameter values.

\section{DisCUSSION AND CONCLUSION}

Using a specific airborne L-band radiometer system featuring kurtosis RFI detection, in campaigns covering a range of target areas spread out over the world from the North Sea to Australia, a unique data set for RFI assessment has been generated. RFI is found everywhere, but the occurrence is highly variable. The most striking feature is that RFI is generally scarce over the ocean and in rural areas where typically a few percent of data are flagged, but it is more dominating over cities, airports, and occasional special installations, where up to $100 \%$ of data may be contaminated. In addition to this uneven distribution in space, there are also large variations over time: Covering the same area on different days reveals special days with much enhanced RFI, even over generally quiet areas like the ocean. Finally, there are also significant regional differences: Southern France/northern Spain has proven to be particularly plagued by RFI.

The radiometer system features very fast sampling and a high-data-rate $(1.8 \mu \mathrm{s})$ output, and using this information on kurtosis-flagged data, the exact amount of RFI contribution to the brightness temperature can be assessed and hence mitigated (for the very often seen pulselike RFI). Using such a procedure, it has been found that the kurtosis method, in addition to flagging harmful RFI, also flags minute RFI spikes that, in the end, would not contribute to the brightness temperature with any significant level. In general, such "false alarms" account for about $50 \%$ of the flagged data, but since only a few percent of data are generally flagged over the target areas, this "overkill" is not of concern for scientific campaign work. At the same time, it must be emphasized that the kurtosis method has its unique features and drawbacks. A sinusoidal signal pulsed with $50 \%$ duty cycle - a signal that appears as $50 \%$ over the time window used in the kurtosis calculation-will not be detected. Thus, a $T_{B}$ measurement, which has been cleaned using the kurtosis method, may still have some RFI contamination. The experience is, however, that kurtosis, in most cases, does a good job. 
The radiometer system is fully polarimetric, so the possible value of using the third and fourth Stokes parameters as RFI indicators can be assessed: Natural-target emission is expected to result in very low values, while RFI is not. Investigations of a few cases reveal some potential.

Analysis of the kurtosis method-false alarms, missed detections, and how these depend on the time over which kurtosis is calculated-is found in [9] and is also ongoing concerning the present data set. Also, the third and fourth Stokes methods, plus combinations of these and other methods, are being analyzed.

Finally, a few considerations about how forthcoming spaceborne L-band radiometer missions can cope with the RFI situation are warranted. SMOS and Aquarius were designed years ago, and relatively classical methods of RFI detection are planned: Basically, look for unusually large brightness temperatures. In addition to this, SMOS, being a polarimetric interferometer, offers some unique possibilities: inspection of the third and fourth Stokes parameters, look for unusual incidence-angle signatures, and look for unusual signals in the long baselines. One challenge for SMOS is that data are only accessible after a substantial integration time of $1.2 \mathrm{~s}$. Hence, RFI spikes are smeared out in time and thus difficult to detect, and if mitigation is carried out, a whole footprint must be discarded. Aquarius uses shorter integration time, and data are available on ground every $10 \mathrm{~ms}$. This facilitates detection of pulselike signals, and only a part of the data corresponding to one footprint may have to be discarded.

Considering space missions a bit further out in the future, new possibilities are obvious. It is important to use the highest possible sampling and downlink rate and calculate kurtosis-much like described for the EMIRAD-2 system discussed in this paper. Some might be frightened by the thought of having fast and substantial digital sampling and processing in a spaceborne system. However, as pointed out in [14], kurtosis can actually be calculated without digital processing using a relatively straightforward double-detector system (basically two classical diode detectors in series). Thus, there is a wealth of possibilities for future missions-and it is interesting to note that we can consider SMOS as the first global spaceborne L-band RFI detection mission. The results are eagerly awaited.

\section{ACKNOWLEDGMENT}

The authors would like to thank Helsinki University of Technology for providing and operating the aircraft, with S. Tauriainen as an extremely dedicated pilot; the SMOS Science Advisory Group for the fruitful discussion; and ESA staff C. Bouzinac and P. Wursteisen for organizing the airborne campaigns.

\section{REFERENCES}

[1] H. M. J. P. Barré, B. Duesmann, and Y. Kerr, "SMOS: The mission and the system," IEEE Trans. Geosci. Remote Sens., vol. 46, no. 3, pp. 587-593, Mar. 2008

[2] D. M. Le Vine, G. Lagerloef, F. R. Colomb, S. H. Yueh, and F. A. Pellerano, "Aquarius: An instrument to monitor sea surface salinity from space," IEEE Trans. Geosci. Remote Sens., vol. 45, no. 7, pp. 20402050, Jul. 2007.

[3] E. G. Njoku, P. Ashcroft, T. K. Chan, and L. Li, "Global survey and statistics of radio-frequency interference in AMSR-E land observations," IEEE Trans. Geosci. Remote Sens., vol. 43, no. 5, pp. 938-947, May 2005.
[4] J. R. Piepmeier and F. A. Pellerano, "Mitigation of terrestrial radar interference in L-band spaceborne microwave radiometers," in Proc. IGARSS, Denver, CO, 2006, pp. 2292-2296.

[5] D. M. Le Vine and M. Haken, "RFI at L-band in synthetic aperture radiometers," in Proc. IGARSS, Toulouse, France, Jul. 21-25, 2003, vol. 3, pp. $1742-1744$.

[6] N. Niamsuwan, J. T. Johnson, and S. W. Ellingson, "Examination of a simple pulse-blanking technique for radio frequency interference mitigation," Radio Sci., vol. 40, no. 5, p. RS5 S03, Jun. 2005.

[7] B. Guner, J. T. Johnson, and N. Niamsuwan, "Time and frequency blanking for radio-frequency interference mitigation in microwave radiometry," IEEE Trans. Geosci. Remote Sens., vol. 45, no. 11, pp. 3672-3679, Nov. 2007.

[8] C. S. Ruf, S. M. Gross, and S. Misra, "RFI detection and mitigation for microwave radiometry with an agile digital detector," IEEE Trans. Geosci. Remote Sens., vol. 44, no. 3, pp. 694-706, Mar. 2006.

[9] R. D. De Roo, S. Misra, and C. S. Ruf, "Sensitivity of the kurtosis statistic as a detector of pulsed sinusoidal RFI," IEEE Trans. Geosci. Remote Sens., vol. 45, no. 7, pp. 1938-1946, Jul. 2007.

[10] J. F. Kenney and E. S. Keeping, "Kurtosis," in Mathematics of Statistics, 3rd ed. Princeton, NJ: Van Nostrand, 1962, ch. 1, sec. 7.12, pp. 102-103.

[11] R. D. De Roo and S. Misra, "A demonstration of the effects of digitization on the calculation of kurtosis for the detection of RFI in microwave radiometry," IEEE Trans. Geosci. Remote Sens., vol. 46, no. 10, pp. 3129 3136, Oct. 2008.

[12] N. Skou, S. S. Søbjærg, J. Balling, and S. S. Kristensen, "A second generation L-band digital radiometer for sea salinity campaigns," in Proc. IGARSS, Denver, CO, Jul. 2006, pp. 3984-3987.

[13] S. Misra, S. S. Kristensen, S. S. Søbjærg, and N. Skou, "CoSMOS: Performance of kurtosis algorithm for radio frequency interference detection and mitigation," in Proc. IGARSS, Barcelona, Spain, Jul. 23-27, 2007, pp. 2714-2717.

[14] J. R. Piepmeier, P. N. Mohammed, and J. J. Knuble, "A double detector for RFI mitigation in microwave radiometers," IEEE Trans. Geosci. Remote Sens., vol. 46, no. 2, pp. 458-465, Feb. 2008.

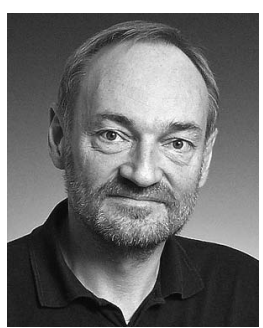

Niels Skou (S'78-M'79-SM'96-F'03) received the M.Sc., Ph.D., and D.Sc. degrees from the Technical University of Denmark (DTU), Lyngby, Denmark, in 1972, 1981, and 1990, respectively.

$\mathrm{He}$ is currently a Professor with DTU, where he is also with the National Space Institute. His research has been directed toward microwave remotesensing systems. After working for three years on the development of radar systems for measuring the ice sheets in Greenland and Antarctica, his interest turned toward microwave radiometry. He developed a scanning, multifrequency, and airborne radiometer system. After that, his subjects were radiometer measurements of sea ice and oil pollution in the sea, spaceborne radiometer systems, and development of new systems for specific purposes. In the mid-1980s, his interest turned back to active instruments, and he became engaged in the development of an airborne, multifrequency, polarimetric, and interferometric synthetic aperture radar system - with special emphasis on calibration fidelity. However, his activity within microwave radiometry has continued, mainly within the areas of synthetic aperture radiometry and polarimetric radiometry. The work on synthetic aperture radiometry has led to the SMOS mission, one of the European Space Agency's Earth Explorer Opportunity Missions. He is a member of the SMOS Science Advisory Group. In support of SMOS, a range of airborne campaigns with L-band radiometers have been carried out. He has taken a special interest in radio-frequency interference, using digital signal processing to detect and mitigate harmful radio-frequency interference. 


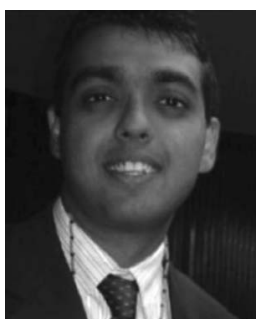

Sidharth Misra received the B.E. degree in electronics and communication engineering from the Nirma Institute of Technology, Gujarat University, Ahmedabad, India, in 2004 and the M.S. degree in electrical engineering and computer science from the University of Michigan, Ann Arbor, in 2006. $\mathrm{He}$ is currently working toward the Ph.D. degree in the Department of Atmospheric, Oceanic, and Space Sciences, University of Michigan.

He was a Research Engineer with the Space Physics Research Laboratory, University of Michigan, where he worked on the analysis and implementation of an agile digital receiver for radio-frequency-interference (RFI) mitigation. He has also worked on Oceansat-II with the Space Applications Centre, Indian Space Research Organisation, Ahmedabad. He was a Research Assistant with the Danish National Space Center, Technical University of Denmark (DTU), Lyngby, performing RFI analysis for CoSMOS, which is an airborne campaign preparing for the SMOS mission. His research interests include microwave radiometry, signal detection and estimation, and image processing.

Dr. Misra was the recipient of the IGARSS 2006 Symposium Prize Paper Award and the IGARSS 2009 Student Paper Award-First Prize.

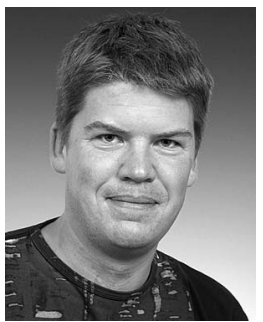

Jan E. Balling received the M.Sc. degree from the Technical University of Denmark (DTU), Lyngby, Denmark, in 2002.

$\mathrm{He}$ is currently with the Division of Microwaves and Remote Sensing, National Space Institute, DTU. His work has mainly been focused on the development of commercial off-the-shelf-based data acquisition and control systems that are suitable for various remote-sensing applications, such as the EMIRAD L-band polarimetric radiometer. In preparation for the European Space Agency's SMOS mission, he has participated in several airborne campaigns and has been involved in postcampaign data calibration and quality-assessment activities. This has led to his special interest being taken in the field of detection and mitigation of harmful RF interference.

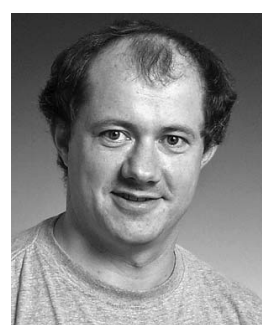

Steen Savstrup Kristensen received the M.Sc. degree in electrical engineering from the Technical University of Denmark (DTU), Lyngby, Denmark, in 1985.

$\mathrm{He}$ is currently with the Division of Microwaves and Remote Sensing, National Space Institute, DTU. His work has mainly been focused on the development and operation of radars and radiometers at the National Space Institute, such as the EMIRAD L-band radiometer, the POLARIS ice sounder, and the $60-\mathrm{MHz}$ ice sounder. He has participated in several ice-sounding campaigns in Greenland and radiometer campaigns in Europe.

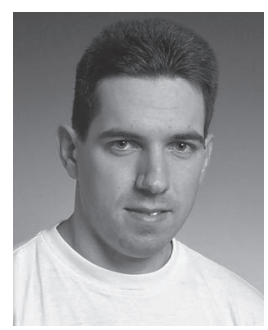

Sten Schmidl Søbjærg (M'99) received the M.Sc.E.E. and Ph.D. degrees from the Technical University of Denmark (DTU), Lyngby, Denmark, in 1996 and 2003, respectively.

$\mathrm{He}$ is currently an Associate Professor with the Division of Microwaves and Remote Sensing, National Space Institute, DTU. His research includes microwave radiometers and their applications. In 1996, he participated in the development of an airborne fully polarimetric dual-frequency scanning radiometer system for 16 and $34 \mathrm{GHz}$, operated on a C-130 aircraft in order to determine the wind direction over the ocean. Since 2001, he has been working on the EMIRAD L-band polarimetric radiometer featuring subharmonic sampling, and he has participated in several airborne campaigns in preparation for the European Space Agency's SMOS mission. $\mathrm{He}$ is currently preparing for SMOS calibration and validation campaigns. His special interest is devoted to accurate instrument calibration and data-quality assessment, including detection and mitigation of RF interference. 